\title{
Prediction of Methotrexate Intolerance in Juvenile Idiopathic Arthritis: a prospective, observational cohort study
}

Evert Hendrik Pieter van Dijkhuizen ${ }^{1,5^{*}}$, Maja Bulatović Ćalasan ${ }^{1}$, Saskia MF Pluijm², Maurits CFJ de Rotte ${ }^{3}$, Sebastiaan J Vastert ${ }^{1}$, Sylvia Kamphuis ${ }^{4}$, Robert de Jonge ${ }^{3}$ and Nico M Wulffraat ${ }^{1}$

\begin{abstract}
Background: Methotrexate (MTX) is an effective and safe drug in the treatment of juvenile idiopathic arthritis (JIA). Despite its safety, MTX-related gastrointestinal adverse effects before and after MTX administration, termed MTX intolerance, occur frequently, leading to non-compliance and potentially premature MTX termination. The aim of this study was to construct a risk model to predict MTX intolerance.

Methods: In a prospective JIA cohort, clinical variables and single nucleotide polymorphisms were determined at MTX start. The Methotrexate Intolerance Severity Score was employed to measure MTX intolerance in the first year of treatment. MTX intolerance was most prevalent at 6 or 12 months after MTX start, which was defined as the outcome for the prediction model. The model was developed in 152 patients using multivariable logistic regression analysis and subsequently internally validated using bootstrapping.

Results: The prediction model included the following predictors: JIA category, antinuclear antibody, parent/patient assessment of pain, Juvenile Arthritis Disease Activity Score-27, thrombocytes, alanine aminotransferase and creatinine. The model classified $77.5 \%$ of patients correctly, and $66.7 \%$ of patients after internal validation by bootstrapping. The lowest predicted risk of MTX intolerance was $18.9 \%$ and the highest predicted risk was $85.9 \%$. The prediction model was transformed into a risk score (range $0-17$ ). At a cut-off of $\geq 6$, sensitivity was $82.0 \%$, specificity $56.1 \%$, positive predictive value was $58.7 \%$ and negative predictive value $80.4 \%$.
\end{abstract}

Conclusions: This clinical prediction model showed moderate predictive power to detect MTX intolerance. To develop into a clinically usable tool, it should be validated in an independent cohort and updated with new predictors. Such an easy-to-use tool could then assist clinicians in identifying patients at risk to develop MTX intolerance, and in turn to monitor them closely and intervene timely in order to prevent the development of MTX intolerance.

Trial registration: ISRCTN register, www.isrctn.com, ISRCTN13524271

Keywords: Juvenile idiopathic arthritis, Methotrexate, Adverse events, Methotrexate intolerance, Prediction model, Predictor

\footnotetext{
* Correspondence: E.H.P.Dijkhuizen@umcutrecht.nl

${ }^{1}$ Department of Paediatric Immunology, University Medical Centre Utrecht,

Wilhelmina Children's Hospital, Utrecht, The Netherlands

${ }^{5}$ Pediatria II, Reumatologia, IRCCS G. Gaslini, Largo Gaslini 5, 16147 Genova,

Italy

Full list of author information is available at the end of the article
}

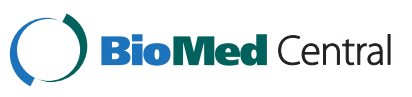

(C) 2015 van Dijkhuizen et al. ; licensee BioMed Central. This is an Open Access article distributed under the terms of the Creative Commons Attribution License (http://creativecommons.org/licenses/by/4.0), which permits unrestricted use, distribution, and reproduction in any medium, provided the original work is properly credited. The Creative Commons Public Domain Dedication waiver (http://creativecommons.org/publicdomain/zero/1.0/) applies to the data made available in this article, unless otherwise stated. 


\section{Background}

Juvenile idiopathic arthritis (JIA) is the most common childhood rheumatic disease [1,2]. In JIA, methotrexate (MTX) is the cornerstone treatment, due to its efficacy and safety. Serious adverse effects such as hepatotoxicity and bone marrow suppression occur rarely [3]. In contrast, MTX-related gastrointestinal adverse effects, such as nausea, abdominal pain and vomiting, occur frequently [4-10]. Folic acid supplementation is an accepted strategy to prevent and treat these adverse effects [11-13]. Despite folic acid use, many JIA patients experience gastrointestinal adverse effects after MTX intake [4-10]. JIA patients also experience anticipatory adverse effects, occurring before MTX administration (at the sight of MTX), and associative adverse effects, occurring when thinking of MTX administration (its colour or smell) $[4,5,14]$. These adverse effects are thought to be a result of classical conditioning to the abovementioned physical symptoms experienced after MTX intake [14]. Importantly, if physical symptoms are absent, conditioned responses cannot develop [15]. Such a combination of symptoms, which we previously termed MTX intolerance, [14] is a significant burden for JIA patients and their parents. Notably, MTX intolerance occurs in up to half of JIA patients on MTX, [14] and can negatively affect their quality of life [6]. Moreover, over three-quarters of intolerant patients reluctantly used or even refused MTX, [14] which, besides leading to non-compliance, could lead to premature discontinuation of MTX, and even replacement by costly biologicals $[5,16,17]$. Such consequences could be avoided, if the development of MTX intolerance is prevented.

To prevent MTX intolerance, it is crucial to predict which patients starting MTX will be at risk to develop it. Thus, clinicians could be able to prevent MTX intolerance in patients at risk by immediate treatment of emerging physical symptoms, which otherwise could give rise to conditioned responses. Treatment of physical symptoms could include lowering the MTX dose, [4] or starting behavioural therapy [5] or anti-emetics [18]. Predicting MTX intolerance would enable clinicians to apply such treatment strategies only in those patients who are likely to develop MTX intolerance.

Single nucleotide polymorphisms (SNPs) involved in the MTX metabolic pathways, and clinical predictors have been associated with MTX-related gastrointestinal adverse effects in rheumatoid arthritis (RA) [19-28] and JIA, the latter of which were reviewed recently [29]. However, to date no model has been constructed to predict MTX intolerance in JIA. The aim of this cohort study was to develop and internally validate such a prediction model, using clinical and genetic predictors.

\section{Methods}

\section{Patients and study design}

An investigator-initiated observational prospective study on efficacy and adverse effects of MTX in patients starting MTX (ISRCTN13524271) was performed at the University Medical Centre Utrecht and Erasmus University Medical Centre Rotterdam, The Netherlands, between January 2008 and October 2012. It was approved by the Ethics Committees of the participating centres and the Central Committee on Research involving Human Subjects, and was conducted according to good clinical practice guidelines.

Patients aged 1-18 years, with a confirmed diagnosis of JIA according to International League of Associations for Rheumatology (ILAR) criteria, [30] who started MTX, were included. Those who had stopped MTX for at least three months, but re-started MTX due to a relapse, were also included. At the time of MTX start, their clinical data (Table 1) were documented in case report forms and blood for the analysis of SNPs was drawn.

All patients completed the previously developed and validated MTX Intolerance Severity Score (MISS) at 3, 6 and 12 months after MTX start [14]. This questionnaire consists of 12 questions, assessing abdominal pain, nausea and vomiting after or before (anticipatory) MTX intake and when thinking of MTX (associative). Furthermore, it assesses behavioural complaints associated with MTX intake, such as crying, restlessness, irritability and refusal to take the drug. The score ranges from 0 to 36 and those with a score of $\geq 6$, including at least one anticipatory, associative or behavioural symptom, were defined as MTX intolerant [14].

\section{Development of MTX intolerance over time and patient selection}

To define the outcome for the prediction model, the development of MTX intolerance at 3, 6 and 12 months after MTX start was assessed. For this analysis, of 175 patients starting MTX treatment, 8 patients were excluded due to a diagnosis other than JIA ( $\mathrm{n}=4$ : Lyme disease, colitis, sarcoidosis, 22q11 deletion syndrome) and use of biologicals at MTX start $(\mathrm{n}=3$ : anakinra; $\mathrm{n}=1$ : etanercept), resulting in 167 eligible patients (Figure 1). Additionally, 25 patients who completed only one MISS during followup were excluded, as their development of MTX intolerance could not be determined. Therefore, the development of MTX intolerance was assessed in 142 patients (Figure 1). In the first year after MTX start, 59 (41.5\%) patients were intolerant (score $\geq 6$ with at least one anticipatory, associative or behavioural complaint) (Table 2). At 3 months, 22 (15.7\%) patients were intolerant. However, intolerance resolved in the majority of these (13 [59.1\%]) at 6 months. At 6 months, the number of intolerant patients increased to 
Table 1 Prevalence, univariable ORs $(95 \%-\mathrm{Cl})$ and p-values for potential predictors of MTX intolerance at MTX start

\begin{tabular}{|c|c|c|c|c|}
\hline \multirow{2}{*}{ Variables } & & \multicolumn{3}{|l|}{ Cohort, $n=152$} \\
\hline & & Frequency $\mathrm{n}(\%)^{\mathrm{a}}$ & OR $(95 \%-\mathrm{Cl})$ & $\mathrm{p}$-value \\
\hline \multicolumn{5}{|l|}{ Demographics } \\
\hline Female & & $92(60.5)$ & $1.34(0.64-2.82)$ & 0.432 \\
\hline Age at disease onset & $>8$ years & $80(52.6)$ & $0.68(0.34-1.36)$ & 0.271 \\
\hline Age at MTX start ${ }^{*}$ & $>12$ years & $72(47.4)$ & $0.54(0.27-1.07)$ & 0.073 \\
\hline Disease duration at MTX start & $>0.5$ years & $103(67.8)$ & $0.79(0.37-1.70)$ & 0.535 \\
\hline \multicolumn{5}{|l|}{$J I A$ category ${ }^{* b}$} \\
\hline Oligoarticular (persistent/extended) & & $62(40.8)$ & Reference & 0.094 \\
\hline Polyarticular (RF negative/positive) & & $64(42.1)$ & $1.91(0.86-4.24)$ & \\
\hline Other (systemic/psoriatic/enthesitis) & & $26(17.1)$ & $0.78(0.27-2.31)$ & \\
\hline \multicolumn{5}{|l|}{ Disease characteristics } \\
\hline $\mathrm{ANA}^{* \mathbf{b}, \mathbf{c}}$ & Positive & $84(55.3)$ & $1.98(0.97-4.07)$ & 0.057 \\
\hline $\mathrm{RF}^{\mathrm{c}}$ & Positive & $16(10.5)$ & $1.52(0.62-3.72)$ & 0.352 \\
\hline $\mathrm{HLA}-\mathrm{B} 27^{\mathrm{c}}$ & Positive & $11(7.2)$ & $0.78(0.29-2.12)$ & 0.510 \\
\hline Uveitis & Present & $21(13.8)$ & $1.44(0.55-3.78)$ & 0.455 \\
\hline \multicolumn{5}{|l|}{ Disease activity } \\
\hline \multirow[t]{3}{*}{ CHAQ disability score ${ }^{c}$} & $\leq 0.250$ & $36(23.7)$ & Reference & 0.395 \\
\hline & $0.250-1.875$ & $88(57.9)$ & $0.61(0.24-1.55)$ & \\
\hline & $>1.875$ & $15(9.9)$ & $0.72(0.18-2.80)$ & \\
\hline \multirow[t]{3}{*}{ Parent/patient assessment of pain ${ }^{\mathbf{k}, \mathbf{c}}$} & $\leq 3 \mathrm{~cm}$ & $58(38.2)$ & Reference & 0.086 \\
\hline & $3-6 \mathrm{~cm}$ & $36(23.7)$ & $2.19(0.84-5.67)$ & \\
\hline & $>6 \mathrm{~cm}$ & $42(27.6)$ & $0.78(0.30-2.02)$ & \\
\hline Parent/patient global assessment ${ }^{c}$ & $>2.5 \mathrm{~cm}$ & $90(59.2)$ & $0.79(0.36-1.72)$ & 0.494 \\
\hline Active joints $^{*}$ & $>2$ & $92(60.5)$ & $2.00(0.91-4.41)$ & 0.070 \\
\hline Limited joints $^{*}$ & $>1$ & $108(71.1)$ & $2.02(0.92-4.46)$ & 0.072 \\
\hline \multirow[t]{3}{*}{$P G A^{d}$} & $\leq 2 \mathrm{~cm}$ & $50(32.9)$ & Reference & 0.496 \\
\hline & $2-5 \mathrm{~cm}$ & $86(56.6)$ & $1.35(0.53-3.47)$ & \\
\hline & $>5 \mathrm{~cm}$ & $16(10.5)$ & $0.87(0.21-3.60)$ & \\
\hline $\mathrm{ESR}^{c}$ & $>15 \mathrm{~mm} / \mathrm{hr}$ & $74(48.7)$ & $1.46(0.66-3.25)$ & 0.341 \\
\hline CRPc & $>10 \mathrm{mg} / \mathrm{L}$ & $49(32.2)$ & $0.83(0.40-1.74)$ & 0.544 \\
\hline \multirow[t]{3}{*}{ JADAS- $27^{*} \mathbf{b}, \mathbf{c}$} & $\leq 5$ & $16(10.5)$ & Reference & 0.048 \\
\hline & $5-15$ & $59(38.8)$ & $0.40(0.11-1.40)$ & \\
\hline & $>15$ & $52(34.2)$ & $0.93(0.25-3.44)$ & \\
\hline \multicolumn{5}{|l|}{ Biochemical variables $^{c}$} \\
\hline Haemoglobin & $>7.5 \mathrm{mmol} / \mathrm{L}$ & $78(51.3)$ & $1.18(0.60-2.32)$ & 0.620 \\
\hline Leucocytes & $>7 \times 10^{9} / \mathrm{L}$ & $96(63.2)$ & $1.21(0.59-2.47)$ & 0.606 \\
\hline Thrombocytes ${ }^{* b}$ & $>350 \times 10^{9} / \mathrm{L}$ & $74(48.7)$ & $1.61(0.82-3.16)$ & 0.161 \\
\hline AST & $>17 \mathrm{IU} / \mathrm{L}$ & $96(63.2)$ & $1.08(0.50-2.36)$ & 0.635 \\
\hline$A L T^{* b}$ & $>12 \mathrm{IU} / \mathrm{L}$ & $101(66.4)$ & $0.41(0.19-0.88)$ & 0.019 \\
\hline Creatinine $^{* \mathbf{b}}$ & $>50 \mu \mathrm{mol} / \mathrm{L}$ & $56(36.8)$ & $0.51(0.24-1.08)$ & 0.069 \\
\hline \multicolumn{5}{|l|}{ Medication } \\
\hline MTX dose, median (IQR) & $\mathrm{mg} / \mathrm{m}^{2} /$ week & $9.9(9.0-11.2)$ & NA & \\
\hline MTX route & oral & $148(97.4)$ & NA & \\
\hline MTX restarted & & $31(20.4)$ & $1.22(0.48-3.11)$ & 0.554 \\
\hline
\end{tabular}


Table 1 Prevalence, univariable ORs $(95 \%-\mathrm{Cl})$ and p-values for potential predictors of MTX intolerance at MTX start (Continued)

\begin{tabular}{|c|c|c|c|c|}
\hline Folic acid & & $150(98.7)$ & NA & \\
\hline Anti-emetics & & $5(3.3)$ & NA & \\
\hline NSAID & & $120(78.9)$ & $0.93(0.38-2.28)$ & 0.655 \\
\hline \multicolumn{5}{|l|}{ Single nucleotide polymorphisms ${ }^{c}$} \\
\hline MTHFR rs1801133 C> T & $\pi$ & $15(9.9)$ & $0.60(0.21-1.69)$ & 0.322 \\
\hline MTHFR rs1801131 A >C & $\mathrm{CC} / \mathrm{AC}$ & $79(52.0)$ & $1.65(0.76-3.62)$ & 0.201 \\
\hline MTRR rs1801394 A > $\mathrm{G}^{*}$ & $\mathrm{GG} / \mathrm{AG}$ & $117(77.0)$ & $0.53(0.24-1.20)$ & 0.123 \\
\hline RFC/SLC19A1 rs1051266 C > T & $\pi$ & $17(11.2)$ & $1.77(0.74-4.25)$ & 0.194 \\
\hline ITPA rs1127354 C > A & $\mathrm{AA} / \mathrm{CA}$ & $15(9.9)$ & $0.62(0.22-1.74)$ & 0.350 \\
\hline AMPD1 rs17602729 G > A & $A A / G A$ & $41(27.0)$ & $1.46(0.70-3.05)$ & 0.304 \\
\hline AT/C rs 2372536 C > G & $\mathrm{GG} / \mathrm{CG}$ & $93(61.2)$ & $0.84(0.39-1.83)$ & 0.614 \\
\hline ADA22 rs73598374 C > T & $\mathrm{TT} / \mathrm{CT}$ & $13(8.6)$ & NA & \\
\hline ADORA2A rs5751876 C > T & $\pi$ & $28(18.4)$ & $1.54(0.65-3.64)$ & 0.319 \\
\hline MDR-1/ABCB1 rs $128503 \mathrm{G}>\mathrm{A}^{*}$ & AA & $32(21.1)$ & $1.73(0.75-3.98)$ & 0.190 \\
\hline$M D R-1 / A B C B 1$ rs $1045642 \mathrm{G}>\mathrm{A}$ & AA & $44(28.9)$ & $1.40(0.65-3.01)$ & 0.376 \\
\hline MDR-1/ABCB1 rs2032582 C > A/T & $\mathrm{AA} / \mathrm{TT}$ & $24(15.8)$ & $1.51(0.63-3.64)$ & 0.344 \\
\hline MRP-1/ABCC1 rs35592 T >C & $\mathrm{CC} / \mathrm{TC}$ & $52(34.2)$ & $0.79(0.39-1.57)$ & 0.494 \\
\hline MRP-1/ABCC1 rs3784862 A > G & $\mathrm{GG} / \mathrm{AG}$ & $73(48.0)$ & $0.97(0.50-1.91)$ & 0.824 \\
\hline MRP-2/ABCC2 rs4148396 C > T & $\pi$ & $18(11.8)$ & $1.57(0.60-4.08)$ & 0.349 \\
\hline$M R P-2 / A B C C 2$ rs717620 C > T & $\mathrm{TT} / \mathrm{CT}$ & $44(28.9)$ & $0.82(0.37-1.82)$ & 0.626 \\
\hline MRP-3/ABCC3 rs4793665 T >C & $\mathrm{CC} / \mathrm{TC}$ & $92(60.5)$ & $0.73(0.36-1.49)$ & 0.381 \\
\hline$M R P-3 / A B C C 3$ rs3785911 $\mathrm{A}>C^{*}$ & $\mathrm{CC} / \mathrm{AC}$ & $78(51.3)$ & $1.67(0.84-3.32)$ & 0.136 \\
\hline MRP-4/ABCC4 rs868853 T >C & $\mathrm{CC} / \mathrm{TC}$ & $22(14.5)$ & $0.88(0.35-2.18)$ & 0.734 \\
\hline MRP-4/ABCC4 rs $2274407 \mathrm{C}>\mathrm{A}$ & $\mathrm{AA} / \mathrm{CA}$ & $20(13.2)$ & $1.33(0.48-3.73)$ & 0.514 \\
\hline MRP-5/ABCC5 rs $2139560 \mathrm{G}>\mathrm{A}$ & $A A / G A$ & $92(60.5)$ & $1.31(0.64-2.68)$ & 0.450 \\
\hline$B C R P / A B C G 2$ rs13120400 T > C & $\mathrm{CC} / \mathrm{TC}$ & $63(41.4)$ & $0.77(0.38-1.59)$ & 0.470 \\
\hline BCRP/ABCG2 rs2231142 G > T & $\Pi \mathrm{T} / \mathrm{GT}$ & $30(19.7)$ & $0.96(0.42-2.20)$ & 0.744 \\
\hline FPGS rs $4451422 \mathrm{~A}>\mathrm{C}$ & $\mathrm{CC} / \mathrm{AC}$ & $102(67.1)$ & $1.37(0.63-2.94)$ & 0.417 \\
\hline GGH rs10106587 A >C & $\mathrm{CC} / \mathrm{AC}$ & $73(48.0)$ & $1.20(0.59-2.46)$ & 0.508 \\
\hline GGH rs3758149 G > A & $A A / G A$ & $77(50.7)$ & $1.20(0.57-2.55)$ & 0.602 \\
\hline PCFT/SLC46A1 rs2239907 C > T & $\mathrm{TT} / \mathrm{CT}$ & $104(68.4)$ & $1.49(0.69-3.23)$ & 0.306 \\
\hline
\end{tabular}

Abbreviations: ALT, alanine aminotransferase; ANA, antinuclear antibody; AST, asparagine aminotransferase; CHAQ, childhood health assessment questionnaire; $\mathrm{Cl}$ confidence interval; CRP, C-reactive protein; ESR, erythrocyte sedimentation rate; HLA, human leucocyte antigen; IQR, interquartile range; IU, international units; JADAS, juvenile arthritis disease activity score; JIA, juvenile idiopathic arthritis; MICE, multivariate imputation by chained equations; MTX, methotrexate; NSAID, non-steroidal anti-inflammatory drug; OR, odds ratio; PGA, physician global assessment; RF, rheumatoid factor.

*Variables associated with the outcome at $p<0.20$ in the univariable logistic regression analysis. Variables with observed frequencies of $<5$ in the cross-tabulation with the outcome were excluded from the univariable logistic analysis: MTX route, use of folic acid, use of anti-emetics and ADA22 rs73598374.

${ }^{\text {a }}$ requencies are based on observed data, not imputed data.

bJA category, ANA, parent/patient assessment of pain, JADAS-27, thrombocytes, ALT and creatinine were included in the multivariable logistic regression analysis. 'MICE was used to impute missing values in the following variables (percentage of missing values): HLA-B27 (60.5), RF (19.1), JADAS-27 (16.4), CRP (15.8), parent/patient global assessment (11.8), RFC/SLC19A1 rs1051266 (11.8), creatinine (11.2), parent/patient assessment of pain (10.5), CHAQ disability score (8.6), MDR-1/ABCB1 rs2032582 (8.6), ALT (7.9), AST (7.2), ESR (5.3), GGH rs3758149 (4.6), MRP-2/ABCC2 rs717620 (3.9), MRP-4/ABCC4 rs868853 (3.9), MRP-5/ABCC5 rs2139560 (3.9), GGH rs10106587 (3.9), MTHFR rs1801131 (3.3), ATIC rs2372536 (3.3), ADORA2A rs5751876 (3.3), MRP-1/ABCC1 rs3784862 (3.3), MRP-2/ABCC2 rs4148396 (3.3), MRP-3/ABCC3 rs4793665 (3.3), BCRP/ABCG2 rs13120400 (3.3), PCFT/SLC46A1 rs2239907 (3.3), MTHFR rs1801133 (2.6), MTRR rs1801394 (2.6), ITPA rs1127354 (2.6), AMPD1 rs17602729 (2.6), ADA22 rs73598374 (2.6), MDR-1/ABCB1 rs1128503 and rs1045642 (2.6), MRP-1/ABCC1 rs35592 (2.6), MRP-3/ABCC3 rs3785911 (2.6), MRP-4/ABCC4 rs2274407 (2.6), BCRP/ABCG2 rs2231142 (2.6), FPGS rs4451422 (2.6), thrombocytes (2.0), ANA (2.0), hemoglobin (1.3), leucocytes (1.3). dPGA was determined retrospectively by an experienced physician (SJV) in 20 visits (13.2\%).

33 (24.1\%), of whom 24 (72.7\%) were newly intolerant. At 12 months, $14(42.4 \%)$ of those intolerant at 6 months stayed intolerant, whereas 8 had less than 6 points on the
MISS and 11 did not complete it. The total number of intolerant patients at 12 months was 30 (23.3\%), of whom 13 (43.3\%) were newly intolerant (Table 2). 


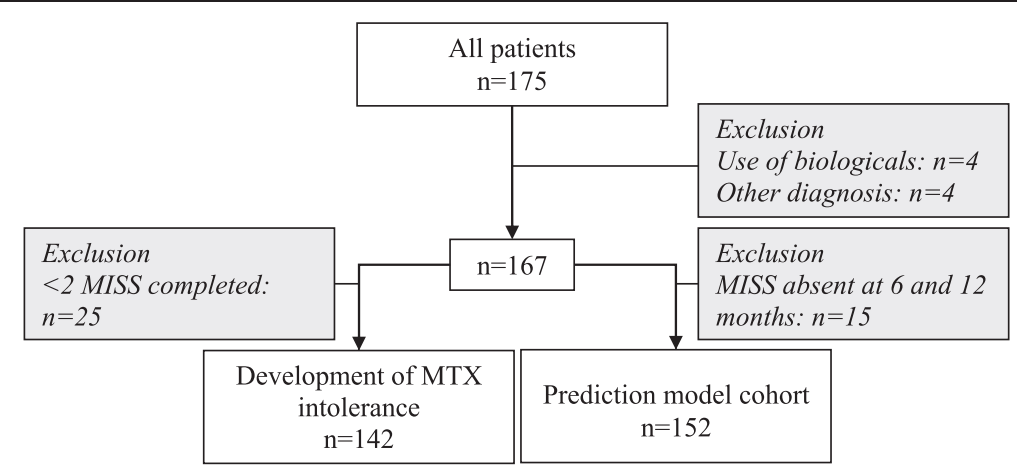

Figure 1 Flowchart. Abbreviations: MISS, Methotrexate Intolerance Severity Score; MTX, methotrexate.

Taken together, the majority of patients developing MTX intolerance did so at 6 or 12 months after MTX start. Consequently, the outcome for the prediction model was defined as MTX intolerance at 6 or 12 months after MTX start.

For the construction of the prediction model, patients with a completed MISS at 6 or 12 months were reselected from the eligible cohort of 167 patients, resulting in 152 included patients (Figure 1).

\section{Potential clinical and genetic predictors}

Potential clinical predictors (demographics, JIA category, disease characteristics, disease activity and biochemical measurements) were identified at baseline (Table 1). Potential genetic predictors were SNPs involved in the MTX metabolic pathways, with a high polymorphic allele frequency and documented functional effects [31]. SNPs were determined in the following genes: methylenetetrahydrofolate reductase (MTHFR), reduced folate carrier (RFC), methionine synthase reductase (MTRR), inosine triphosphatase (ITPA), adenosine monophosphate deaminase (AMPD), aminoimidazole4-carboxamide ribonucleotide transformylase (ATIC), adenosine-deaminase $(A D A)$, adenosine A2A receptor (ADORA2A), multidrug resistance $(M D R) 1$, multidrug resistance protein $(M R P) 1-5$, breast cancer resistance protein (BCRP), folylpolyglutamate synthase (FPGS), gamma glutamyl hydrolase $(G G H)$ and proton-coupled folate transporter (PCFT) (Table 1).

Table 2 MTX intolerance development

\begin{tabular}{lll}
\hline Time point & $\boldsymbol{N}$ & Intolerance, $\mathbf{n}(\%)^{\mathbf{a}}$ \\
\hline 3 months & $140^{\mathbf{b}}$ & $22(15.7)$ \\
6 months & $137^{\mathbf{b}}$ & $33(24.1)$ \\
12 months & $129^{\mathbf{b}}$ & $30(23.3)$ \\
First treatment year & 142 & $59(41.5)$ \\
6 or 12 months $^{\mathbf{d}}$ & $152^{\mathbf{c}}$ & $51(33.6)$ \\
\hline
\end{tabular}

Abbreviations: MTX, methotrexate; $n$, number of patients.

${ }^{\mathbf{a}}$ Frequencies are based on observed data; ${ }^{\mathbf{b}}$ Patients still on MTX; ${ }^{\mathbf{c}}$ Cohort for prediction model construction; ${ }^{\mathbf{d}}$ Outcome was imputed in $21.7 \%$ of cases.

\section{Statistical analysis}

\section{Prediction model construction}

The prediction model was constructed in several steps. First, missing values were imputed using multivariate imputation by chained equations (MICE) [32]. This was done to ensure that all collected data could be used for the development of the model. Second, to facilitate implementation of the model in daily clinical practice, continuous variables were dichotomised or categorised, according to patterns in the data or the risk gradients across percentiles, and the cut-off points with the lowest p-value on the log-likelihood ratio test (i.e. those yielding the optimal association) were chosen [33]. Third, all variables were entered in a univariable logistic regression analysis. The results are presented as regression coefficients $(\beta)$ and odds ratios (OR) with $95 \%$ confidence intervals $(95 \% \mathrm{CI})$. The regression coefficients are an indication of the direction and the magnitude of the effect of the individual predictors, whereas the ORs with 95\% CI indicate the significance of the association.

Variables with a p-value $<0.20$ on the log-likelihood ratio test in the univariable analysis were eligible for inclusion in the multivariable logistic regression analysis. The maximum number of included variables equalled the square root of the number of cases (MTX intolerant patients) in the cohort. If more variables were eligible than the allowed maximum, or if variables correlated (Spearman's $\mid$ rho $\mid>0.40$ ), those with the lowest p-value on the log-likelihood ratio test were included in the multivariable analysis. In addition, presence of effect modification by the predictors in the model was assessed. Effect modification is the situation in which the effect of one predictor on the outcome is modified by the value of another factor. For example, the effect of a predictor may differ between boys and girls. Statistically, this is tested by adding interaction terms to the model, allowing the regression coefficients to take different values for different categories of patients.

Predictive power of the model was assessed with the C-statistic, which reflects the percentage of patients 
classified correctly. To determine whether the model fit the data well, the Hosmer-Lemeshow test was employed. Multicollinearity was tested with variance inflation factors (VIF).

\section{Prediction model validation and risk score computation}

All prediction models need to be validated. Since no independent cohort was available, the model was internally validated using an established statistical technique, called bootstrap [34-36]. In short, 200 bootstrap cohorts (of equal size as the original dataset, $n=152$ ) were randomly drawn, with replacement, from the cases in the original dataset. Next, to each bootstrap cohort, bootstrap multivariable models were fitted (200 in total) using exactly the same methods as described above for the original model, and the corresponding $\mathrm{C}$-statistics $\left(\mathrm{C}_{\text {boot }}\right)$ were determined. Then, the probability of MTX intolerance of the patients in the original dataset was calculated using each of these multivariable models, resulting in another set of C-statistics $\left(\mathrm{C}_{\text {boot-original }}\right)$ reflecting the percentage of patients predicted correctly according to each of these models. The difference between $\mathrm{C}_{\text {boot-original values and }}$ $\mathrm{C}_{\text {boot }}$ values is an estimate of the so-called optimism value (i.e. how much the original model fitted to the original dataset was optimistic compared to the "real" performance of the model in the population). Therefore, in order to obtain the final adjusted C-statistic, indicating the "real" performance of the model in the population, [36] two additional steps need to be performed: a) Subtraction of $\mathrm{C}_{\text {boot-original }}$ values from $\mathrm{C}_{\mathrm{boot}}$ values and averaging them in order to obtain the optimism value; b) Subtraction of this optimism value from $\mathrm{C}_{\text {original }}$ (the C-statistic of the original model, developed in the original dataset), thus obtaining the final adjusted C-statistic. Furthermore, to correct for overfitting, the regression coefficients were reduced with a shrinkage factor, calculated from the bootstrap re-sampling.

All the above mentioned procedures were performed twice. Firstly, only the routinely available clinical variables were considered as potential predictors. Secondly, SNPs were also considered as potential predictors in order to determine whether they contributed to the prediction of MTX intolerance.

To compute a risk score of becoming MTX intolerant, the shrunken regression coefficients were multiplied and rounded off to obtain simple scores that sum up to a total risk score. Sensitivity, specificity, positive predictive value (PPV), negative predictive value (NPV) and accuracy of various cut-off points were calculated.

Statistical analyses were carried out with $\mathrm{R}$ statistics version 2.15.0 (R Foundation for Statistical Computing, Vienna, Austria), using the packages Hmisc (by Frank E Harrell Jr with contributions from many other users, version 3.9-3, 2012) and mice [32].

\section{Results}

Baseline characteristics of the prediction model cohort

The prediction model was constructed in 152 patients. According to the outcome as defined above, 51 (33.6\%) patients were MTX intolerant (Table 2). Intolerant and tolerant patients did not differ regarding the proportion of MTX re-starters, MTX dose, route of administration, concomitant medication use or disease activity (Juvenile Arthritis Disease Activity score [JADAS-27]) at 6 and 12 months after MTX start (data not shown).

Nineteen (12.5\%) patients discontinued MTX treatment during the follow-up, because of MTX intolerance $(n=8)$, disease remission $(n=3)$, insufficient effect $(n=2)$, MTX toxicity (increased liver enzymes: $\mathrm{n}=1$ ) or other reasons $(n=5)$. Patients also switched the route of administration due to gastrointestinal complaints (either from oral to subcutaneous or vice versa): 8 patients after 3 months, 6 patients after 6 months and 1 patient after 12 months.

Baseline characteristics are depicted in Table 1. Thirtyone patients (20.4\%) had re-started MTX treatment due to a relapse after at least three months discontinuation. The majority of patients had either oligoarticular or polyarticular JIA (82.9\%), with high disease activity (median JADAS-27 of 12.7 [interquartile range 7.6-18.2]). Median MTX dose was $9.9 \mathrm{mg} / \mathrm{m}^{2} /$ week, administered mostly as oral MTX (97.4\%) with concomitant use of folic acid (98.7\%).

\section{Clinical prediction model}

First, a model was constructed, containing clinical variables only, excluding the SNPs. Ten clinical variables were associated in the univariable analysis with MTX intolerance $(\mathrm{p}<0.20$; Table 1$)$. The maximum number of variables allowed in the multivariable analysis was seven. Those with the lowest $\mathrm{p}$-value were selected for the clinical prediction model, namely JIA category, JADAS-27, parent/patient assessment of pain, antinuclear antibody (ANA), alanine aminotransferase (ALT), thrombocytes and creatinine, and an interaction term between creatinine and JIA category was added. The C-statistic of the clinical prediction model was $77.5 \%$ (Table 3). The model fit the data well, as shown by a non-significant Hosmer-Lemeshow test $(\mathrm{p}=0.705)$. There was no multicollinearity (data not shown).

\section{Clinical-genetic prediction model}

Next, SNPs were considered as potential predictors in order to determine their contribution to MTX intolerance prediction. Four SNPs in the MTRR, RFC, MDR-1 and $M R P-3$ genes had univariable p-values of $<0.20$, however these p-values (range: 0.123-0.194) were generally higher than those of the clinical model variables (range: 0.0480.161) (Table 1). Hence, since seven variables with the smallest p-values were selected for multivariable analysis, 
Table 3 Prediction model and scores for MTX intolerance

\begin{tabular}{|c|c|c|c|c|c|}
\hline \multicolumn{2}{|l|}{ Predictors } & OR (95\%-Cl) & p-value & $\beta^{a}$ & Score $^{b}$ \\
\hline \multicolumn{6}{|l|}{ JIA category } \\
\hline \multicolumn{2}{|l|}{ Oligoarticular (persistent/extended) } & Reference & & & 0 \\
\hline \multicolumn{2}{|l|}{ Polyarticular (RF negative/positive) } & $4.99(1.36-18.34)$ & 0.016 & 0.914 & 5 \\
\hline \multicolumn{2}{|l|}{ Other (systemic/psoriatic/enthesitis) } & $0.93(0.16-5.49)$ & 0.935 & -0.042 & 0 \\
\hline ANA & Positive & $1.98(0.83-4.68)$ & 0.122 & 0.387 & 2 \\
\hline \multirow[t]{3}{*}{ Parent/patient assessment of pain } & $\leq 3 \mathrm{~cm}$ & Reference & & & 0 \\
\hline & $3-6 \mathrm{~cm}$ & $2.06(0.72-5.89)$ & 0.175 & 0.412 & 2 \\
\hline & $>6 \mathrm{~cm}$ & $0.60(0.17-2.07)$ & 0.421 & -0.288 & -1 \\
\hline \multirow[t]{3}{*}{ JADAS-27 } & $\leq 5$ & Reference & & & 0 \\
\hline & $5-15$ & $0.35(0.08-1.56)$ & 0.168 & -0.599 & -3 \\
\hline & $>15$ & $0.77(0.14-4.32)$ & 0.766 & -0.150 & -1 \\
\hline Thrombocytes & $>350 \times 10^{9} / \mathrm{L}$ & $1.27(0.49-3.27)$ & 0.621 & 0.136 & 1 \\
\hline ALT & $>12 \mathrm{IU} / \mathrm{L}$ & $0.39(0.16-0.96)$ & 0.040 & -0.534 & -3 \\
\hline Creatinine & $>50 \mu \mathrm{mol} / \mathrm{L}$ & $1.37(0.33-5.67)$ & 0.665 & 0.179 & 1 \\
\hline \multicolumn{6}{|l|}{ Interaction term creatinine: JIA category } \\
\hline \multicolumn{2}{|l|}{$>50 \mu \mathrm{mol} / \mathrm{L} \&$ polyarticular arthritis } & $0.17(0.02-1.35)$ & 0.093 & -1.022 & -5 \\
\hline \multicolumn{2}{|l|}{$>50 \mu \mathrm{mol} / \mathrm{L} \&$ other $\mathrm{J} / \mathrm{A}$ category } & $0.82(0.07-9.74)$ & 0.878 & -0.110 & -1 \\
\hline \multicolumn{2}{|l|}{ Constant } & & & -0.039 & 7 \\
\hline \multicolumn{2}{|l|}{ C-statistic } & $77.5 \%$ & & & \\
\hline \multicolumn{2}{|c|}{ C-statistic (optimism-corrected by bootstrap) } & $66.7 \%$ & & & \\
\hline \multicolumn{2}{|l|}{ Hosmer-Lemeshow test (p-value) } & 0.705 & & & \\
\hline
\end{tabular}

Abbreviations: ALT, alanine aminotransferase; ANA, anti-nuclear antibody; Cl, confidence interval; JADAS, juvenile arthritis disease activity score; JIA, juvenile idiopathic arthritis; MTX, methotrexate; OR, odds ratio; RF, rheumatoid factor.

${ }^{\mathrm{a}}$ These are shrunk coefficients (by factor 0.5688 ) to correct for overfitting.

${ }^{\text {b }}$ Shrunk coefficients were multiplied by 5 and rounded off to the nearest integer. The constant was adjusted to obtain the minimum score of 0 .

only the MTRR rs1801394 SNP, next to six clinical variables (those from the abovementioned clinical model, excluding thrombocytes), were included in the model. The model's C-statistic was $77.7 \%$.

\section{Prediction model validation}

Both the clinical and the clinical-genetic prediction model were internally validated using bootstrapping. Upon internal validation, the corrected $\mathrm{C}$-statistic of the clinical model was $66.7 \%$, whereas the corrected C-statistic of the clinical-genetic model was $64.6 \%$.

Since the clinical-genetic model did not perform better than the model with clinical variables, the latter was given preference as clinical variables are readily available at MTX start, making it easier to apply the model in clinical practice.

\section{Risk score}

To enable health care professionals to use the model easily, the shrunken regression coefficients of the clinical model's predictors, transformed into simple scores, were used to compute an individual risk score for being MTX intolerant. This score ranged from 0 to 17 points, with a higher score reflecting a higher probability of MTX intolerance (Table 3). The lowest predicted risk of being MTX intolerant was $18.9 \%$, if the following predictors were present: oligoarticular JIA, negative ANA, parent/ patient assessment of pain $>6 \mathrm{~cm}$, JADAS-27 of 5-15 points, thrombocytes $\leq 350 \times 10^{9} / \mathrm{L}$, ALT $>12$ IU/L and creatinine $\leq 50 \mu \mathrm{mol} / \mathrm{L}$. The combination of these predictors resulted in a score of 0 [7 (the constant) $+0+0+$ $(-1)+(-3)+0+(-3)+0]$ (Table 3). On the other hand, the highest predicted risk of being MTX intolerant was $85.9 \%$, if the following predictors were present: polyarticular JIA, positive ANA, parent/patient assessment of pain of 3-6 cm, JADAS- $27 \leq 5$ points, thrombocytes $>350 \times$ $10^{9} / \mathrm{L}$, ALT $\leq 12 \mathrm{IU} / \mathrm{L}$ and creatinine $\leq 50 \mu \mathrm{mol} / \mathrm{L}$. The combination of these predictors resulted in a score of 17 $[7+5+2+2+0+1+0+0]$.

Within the $0-17$ range, the diagnostic accuracy of different cut-off scores for predicting the risk of being MTX intolerant was evaluated by computing the corresponding sensitivity, specificity, PPV, NPV, and accuracy (Table 4). Our goal was to correctly identify as many future MTX intolerant patients as possible (high sensitivity), while attempting to avoid misidentification of 
Table 4 Diagnostic parameters of the risk score for various cut-off scores

\begin{tabular}{llllll}
\hline Cut-off & Sensitivity (\%) & Specificity (\%) & PPV (\%) & NPV (\%) & Accuracy (\%) \\
\hline$\geq 4$ & 93.4 & 29.9 & 50.3 & 85.7 & 57.3 \\
$\geq 5$ & 87.8 & 46.1 & 55.3 & 83.3 & 64.1 \\
$\geq 6$ & 82.0 & 56.1 & 58.7 & 80.4 & 67.3 \\
$\geq 7$ & 69.2 & 69.9 & 63.6 & 74.9 & 69.6 \\
$\geq 8$ & 58.7 & 80.3 & 69.4 & 71.9 & 71.0 \\
$\geq 9$ & 46.0 & 86.8 & 72.6 & 67.9 & 69.2 \\
\hline
\end{tabular}

Abbreviations: NPV, negative predictive value; PPV, positive predictive value.

tolerant patients as intolerant patients (moderate specificity). This was reached at the cut-off score $\geq 6$, where $82 \%$ of intolerant patients and $56.1 \%$ of tolerant patients were identified correctly.

\section{Discussion}

We developed and internally validated a prediction model for MTX intolerance at 6 or 12 months after MTX start in a large JIA cohort, consisting of routine clinical variables: JIA category, JADAS-27, parent/patient assessment of pain, ANA, ALT, thrombocytes, creatinine and an interaction term between creatinine and JIA category. The model classified $77.5 \%$ of patients correctly, and $66.7 \%$ after internal validation. It should be validated in an independent cohort and updated with other predictors.

In our model, patients who had more pain $(>6 \mathrm{~cm})$, higher baseline disease activity assessed with JADAS-27 and higher ALT, had a lower risk to become MTX intolerant. On the other hand, patients with positive ANA, who had less pain $(3-6 \mathrm{~cm})$, higher thrombocyte levels and higher creatinine, had an increased risk of MTX intolerance. Creatinine level and age were correlated, so creatinin can be regarded as a surrogate marker for age (median age was 7.5 years [patients with creatinine $\leq 50 \mu \mathrm{mol} / \mathrm{L}$ ] versus 13.7 years [creatinine $>50 \mu \mathrm{mol} / \mathrm{L}$ ]). The relationship between JIA category, creatinine (age) and MTX intolerance was complex: In younger patients, polyarticular JIA was a strong predictor for intolerance (score 5, Table 3), whereas in older patients this effect disappeared (score 5 for polyarticular JIA and -5 for the interaction term between older patients (higher creatinine) and polyarticular JIA).

To predict which patients are prone to develop MTX intolerance, our risk score could be readily used by clinicians, since it is based on clinical variables, which are routinely determined and available for all JIA patients before MTX start. At the cut-off score of $\geq 6$, as many as $82 \%$ of intolerant patients were classified correctly (high sensitivity), while maintaining correct classification of $56.1 \%$ of tolerant patients (modest specificity). Table 4 provides the sensitivity and specificity of other potential cut off points.
Identification of patients at risk increases patients' and clinicians' awareness of MTX intolerance. In patients at risk, clinicians should frequently (i.e. every 4 weeks) monitor MTX-related gastrointestinal adverse effects, using the MISS, from the very start of MTX treatment. This would enable clinicians to treat the emerging physical symptoms early, for example by lowering MTX dose, [4] adding anti-emetics [18] or applying behavioural therapy, [5] thus preventing the development of a classical conditioning response [15] and hence MTX intolerance. The effect of these timely interventions on the development of MTX intolerance should be determined in a clinical trial.

The outcome of our prediction model was defined as MTX intolerance at 6 or 12 months after MTX start, since the majority of patients developing MTX intolerance did so at these time-points. The later onset of MTX intolerance is consistent with the notion that the development of MTX intolerance is governed by a classical conditioning response, which worsens over time $[5,14]$. Moreover, in our previous cross-sectional study in patients with longer MTX use (interquartile range: 0.6-3.6 years), we demonstrated higher prevalence of MTX intolerance (50.5-67.5\%) compared to the prevalence of $34.1 \%$ in the present longitudinal study during the first year of MTX treatment [14]. This also supports the notion that MTX intolerance takes time to develop and that longer MTX use may increase the risk of MTX intolerance. To determine whether the risk of MTX intolerance indeed increases with longer MTX use, development of MTX intolerance should be monitored beyond one year of MTX use. Nevertheless, MTX intolerance ensued in $15.8 \%$ of patients already after 3 months of MTX use. Interestingly, patients who had restarted MTX had a higher risk of becoming intolerant after 3 months than those newly starting MTX (36\% versus $12.7 \%, \mathrm{p}=0.015$ ).

To our knowledge, no previous studies have developed a similar model and a corresponding risk score to predict the occurrence of MTX-induced gastrointestinal adverse effects in JIA. In a recently published paper, predictors for MTX adverse events in JIA patients, including the predictors in the current model, were 
reviewed. Only a few candidate predictors were elucidated, and validation of these lacked [29].

Our study did not identify genotypes as predictors for intolerance. In contrast, in RA, two studies identified combinations of risk genotypes to predict adverse effects in general and gastrointestinal adverse effects in particular $[20,26]$. In our study, only 4 of 27 SNPs were moderately associated with MTX intolerance and only one SNP could be included in the clinical-genetic model, which had comparable predictive power as the clinical model. Previously, in RA and JIA, significant associations $(\mathrm{p}<0.05)$ were reported between SNPs in the MTHFR, ATIC, ADORA, MRP2/ABCC2 and GGH genes and gastrointestinal adverse effects [19-22,24-28,37,38]. SNPs in these genes were not associated with MTX intolerance in our study, which could be due to disparities in patient groups (RA versus JIA), cohorts (cross-sectional versus longitudinal), and the definition of MTXinduced gastrointestinal complaints (after MTX versus before and after MTX use). These results taken together with our current study show that it is still difficult to predict reliably the risk of developing MTX adverse events in general and MTX intolerance in particular.

The strengths of our study were that MTX intolerance was assessed using a validated questionnaire. In addition, the model was constructed and internally validated in a large prospective JIA cohort. Internal validation using bootstrapping is an established method to estimate the performance of a prediction model in the population, comparable to external validation in an independent cohort [34-36].

\section{Conclusions}

In conclusion, we developed and internally validated a clinical prediction model for MTX intolerance in a large JIA cohort. It is an easy-to-use tool to identify patients at risk of developing MTX intolerance, and in turn to monitor them closely and intervene timely, in order to prevent MTX intolerance and its negative impact on patients' daily lives, compliance and continuation of an effective treatment. In its current composition, the model performs moderately well and should be validated in an independent cohort and updated with new predictor variables before it can be broadly used in clinical practice.

\section{Competing interests}

The authors declare that they have no competing interests.

\section{Authors' contributions}

PvD collected data, performed statistical analyses and drafted the manuscript. MBC participated in study design, collected data, drafted the manuscript and helped with statistical analyses. SP coordinated statistical analyses and participated in study design. MdR participated in study design and coordinated SNP analyses. SV participated in study design and enrolled patients. SK participated in study design and enrolled patients. RdJ participated in study design and coordination. NW conceived of the study and participated in study design and coordination. All authors read and approved the final manuscript.

\section{Acknowledgements}

We wish to acknowledge: M.J.C. Eijkemans for statistical support; A. Blaauw, M.J.W. van Opdorp and A. van Dijk for valuable assistance during patient inclusion, case report form completion and investigator site file maintenance; and B. van Zelst and P. Griffioen for SNP analysis.

\section{Funding}

This project has received funding from the $7^{\text {th }}$ Framework programme of the EU, SP3-People, support for training and career development for researchers (Marie Curie), Network for Initial Training (ITN), FP7-PEOPLE-2011-ITN, under the Marie Skłodowska-Curie grant agreement No 289903 [grant to EHPvD], SHARE project, EAHC grant number 20111202 [grant to NMW] and the Dutch Arthritis Association [NR 07-01-402 to NMW, NR 06-2-402 to RdJ]. The sponsors had no role in the study planning, design, management or data analysis.

\section{Author details}

${ }^{1}$ Department of Paediatric Immunology, University Medical Centre Utrecht, Wilhelmina Children's Hospital, Utrecht, The Netherlands. 'Department of Paediatric Haemato-Oncology, Erasmus University Medical Centre Rotterdam, Sophia Children's Hospital, Rotterdam, The Netherlands. ${ }^{3}$ Department of Clinical Chemistry, Erasmus University Medical Centre Rotterdam, Rotterdam, The Netherlands. ${ }^{4}$ Department of Paediatric Rheumatology, Erasmus University Medical Centre Rotterdam, Sophia Children's Hospital, Rotterdam, The Netherlands. ${ }^{5}$ Pediatria II, Reumatologia, IRCCS G. Gaslini, Largo Gaslini 5, 16147 Genova, Italy.

Received: 5 September 2014 Accepted: 1 February 2015

Published online: 18 February 2015

\section{References}

1. Prakken B, Albani S, Martini A. Juvenile idiopathic arthritis. Lancet. 2011;377:2138-49.

2. Ravelli A, Martini A. Juvenile idiopathic arthritis. Lancet. 2007;369:767-78.

3. Ortiz-Alvarez O, Morishita K, Avery G, Green J, Petty RE, Tucker LB, et al. Guidelines for blood test monitoring of methotrexate toxicity in juvenile idiopathic arthritis. J Rheumatol. 2004;31:2501-6.

4. Murray KJ, Lovell DJ. Advanced therapy for juvenile arthritis. Best Pract Res Clin Rheumatol. 2002;16:361-78.

5. van der Meer A, Wulffraat NM, Prakken BJ, Gijsbers B, Rademaker CM, Sinnema G. Psychological side effects of MTX treatment in juvenile idiopathic arthritis: a pilot study. Clin Exp Rheumatol. 2007;25:480-5.

6. Brunner HI, Johnson AL, Barron AC, Passo MH, Griffin TA, Graham TB, et al. Gastrointestinal symptoms and their association with health-related quality of life of children with juvenile rheumatoid arthritis: validation of a gastrointestinal symptom questionnaire. J Clin Rheumatol. 2005;11:194-204.

7. Hashkes PJ, Laxer RM. Medical treatment of juvenile idiopathic arthritis. JAMA. 2005;294:1671-84.

8. Ruperto N, Murray K, Gerloni V, Wulffraat N, de Oliveira SK, Falcini F, et al. A randomized trial of parenteral methotrexate comparing an intermediate dose with a higher dose in children with juvenile idiopathic arthritis who failed to respond to standard doses of methotrexate. Arthritis Rheum. 2004:50:2191-201.

9. Ramanan AV, Whitworth P, Baildam EM. Use of methotrexate in juvenile idiopathic arthritis. Arch Dis Child. 2003;88:197-200.

10. Alsufyani K, Ortiz-Alvarez O, Cabral DA, Tucker LB, Petty RE, Malleson PN. The role of subcutaneous administration of methotrexate in children with juvenile idiopathic arthritis who have failed oral methotrexate. J Rheumatol. 2004;31:179-82.

11. Ravelli A, Migliavacca D, Viola S, Ruperto N, Pistorio A, Martini A. Efficacy of folinic acid in reducing methotrexate toxicity in juvenile idiopathic arthritis. Clin Exp Rheumatol. 1999;17:625-7.

12. Alarcon GS, Morgan SL. Folinic acid to prevent side effects of methotrexate in juvenile rheumatoid arthritis. J Rheumatol. 1996;23:2184-5.

13. Hunt PG, Rose CD, Ilvain-Simpson G, Tejani S. The effects of daily intake of folic acid on the efficacy of methotrexate therapy in children with juvenile rheumatoid arthritis. A controlled study. J Rheumatol. 1997;24:2230-2.

14. Bulatovic M, Heijstek MW, Verkaaik M, van Dijkhuizen EHP, Armbrust W, Hoppenreijs EP, et al. High prevalence of methotrexate intolerance in juvenile idiopathic arthritis: development and validation of a methotrexate intolerance severity score. Arthritis Rheum. 2011;63:2007-13. 
15. Figueroa-Moseley C, Jean-Pierre P, Roscoe JA, Ryan JL, Kohli S, Palesh OG, et al. Behavioral interventions in treating anticipatory nausea and vomiting. J Natl Compr Canc Netw. 2007;5:44-50.

16. Beukelman T, Patkar NM, Saag KG, Tolleson-Rinehart S, Cron RQ, DeWitt EM, et al. 2011 American College of Rheumatology recommendations for the treatment of juvenile idiopathic arthritis: initiation and safety monitoring of therapeutic agents for the treatment of arthritis and systemic features. Arthritis Care Res (Hoboken). 2011:63:465-82.

17. Horneff $\mathrm{G}$. Update on biologicals for treatment of juvenile idiopathic arthritis. Expert Opin Biol Ther. 2013;13(3):376-76. doi:10.1517/14712598.2013.735657.

18. Ravelli A, Martini A. Methotrexate in juvenile idiopathic arthritis: answers and questions. J Rheumatol. 2000;27:1830-3.

19. Davila L, Ranganathan P. Pharmacogenetics: implications for therapy in rheumatic diseases. Nat Rev Rheumatol. 2011;7:537-50.

20. Dervieux T, Greenstein N, Kremer J. Pharmacogenomic and metabolic biomarkers in the folate pathway and their association with methotrexate effects during dosage escalation in rheumatoid arthritis. Arthritis Rheum 2006;54:3095-103.

21. Fisher MC, Cronstein BN. Metaanalysis of methylenetetrahydrofolate reductase (MTHFR) polymorphisms affecting methotrexate toxicity. J Rheumatol. 2009;36:539-45.

22. Hider SL, Thomson W, Mack LF, Armstrong DJ, Shadforth M, Bruce IN. Polymorphisms within the adenosine receptor 2a gene are associated with adverse events in RA patients treated with MTX. Rheumatology (Oxford). 2008:47:1156-9.

23. Malik F, Ranganathan P. Methotrexate pharmacogenetics in rheumatoid arthritis: a status report. Pharmacogenomics. 2013;14:305-14.

24. Ranganathan P, Culverhouse R, Marsh S, Mody A, Scott-Horton TJ, Brasington $R$, et al. Methotrexate (MTX) pathway gene polymorphisms and their effects on MTX toxicity in Caucasian and African American patients with rheumatoid arthritis. J Rheumatol. 2008;35:572-9.

25. Stamp LK, Chapman PT, O'Donnell JL, Zhang M, James J, Frampton C, et al. Polymorphisms within the folate pathway predict folate concentrations but are not associated with disease activity in rheumatoid arthritis patients on methotrexate. Pharmacogenet Genomics. 2010;20:367-76.

26. Weisman MH, Furst DE, Park GS, Kremer JM, Smith KM, Wallace DJ, et al. Risk genotypes in folate-dependent enzymes and their association with methotrexate-related side effects in rheumatoid arthritis. Arthritis Rheum. 2006;54:607-12.

27. Wessels JA, Kooloos WM, De JR, De Vries-Bouwstra JK, Allaart CF, Linssen A et al. Relationship between genetic variants in the adenosine pathway and outcome of methotrexate treatment in patients with recent-onset rheumatoid arthritis. Arthritis Rheum. 2006;54:2830-9.

28. Wessels JA, De Vries-Bouwstra JK, Heijmans BT, Slagboom PE, Goekoop-Ruiterman YP, Allaart CF, et al. Efficacy and toxicity of methotrexate in early rheumatoid arthritis are associated with single-nucleotide polymorphisms in genes coding for folate pathway enzymes. Arthritis Rheum. 2006;54:1087-95.

29. van Dijkhuizen EP, Wulffraat NM. Prediction of methotrexate efficacy and adverse events in patients with juvenile idiopathic arthritis: a systematic literature review. Pediatr Rheumatol Online J. 2014;12:51.

30. Petty RE, Southwood TR, Manners P, Baum J, Glass DN, Goldenberg J, et al. International League of Associations for Rheumatology classification of juvenile idiopathic arthritis: second revision, Edmonton, 2001. J Rheumatol. 2004:31:390-2

31. Bulatovic M, Heijstek MW, van Dijkhuizen EH, Wulffraat NM, Pluijm SM, De $J R$. Prediction of clinical non-response to methotrexate treatment in juvenile idiopathic arthritis. Ann Rheum Dis. 2012;71:1484-9.

32. Van Buuren S, Groothuis-Oudshoorn K. MICE: Multivariate Imputation by Chained Equations in R. J Stat Soft. 2011;45:1-67.

33. Hosmer DW, Lemeshow S. Applied Logistic Regression. New York: John Wiley \& Sons; 1989.

34. Harrell Jr FE, Lee KL, Mark DB. Multivariable prognostic models: issues in developing models, evaluating assumptions and adequacy, and measuring and reducing errors. Stat Med. 1996;15:361-87.

35. Steyerberg EW, Harrell Jr FE, Borsboom GJJM, Eijkemans MJC, Vergouwe Y, Habbema JD. Internal validation of predictive models: Efficiency of some procedures for logistic regression analysis. J Clin Epidemiol. 2001;54:774-81.

36. Steyerberg EW. Clinical Prediction Models: A Practical Approach to Development, Validation, and Updating. New York: Springer; 2009.
37. Schmeling H, Biber D, Heins S, Horneff G. Influence of methylenetetrahydrofolate reductase polymorphisms on efficacy and toxicity of methotrexate in patients with juvenile idiopathic arthritis. J Rheumatol. 2005;32:1832-6.

38. Tukova J, Chladek J, Hroch M, Nemcova D, Hoza J, Dolezalova P. 677TT genotype is associated with elevated risk of methotrexate (MTX) toxicity in juvenile idiopathic arthritis: treatment outcome, erythrocyte concentrations of MTX and folates, and MTHFR polymorphisms. J Rheumatol. 2010;37:2180-6.

\section{Submit your next manuscript to BioMed Central and take full advantage of:}

- Convenient online submission

- Thorough peer review

- No space constraints or color figure charges

- Immediate publication on acceptance

- Inclusion in PubMed, CAS, Scopus and Google Scholar

- Research which is freely available for redistribution 